\title{
PedsQL for Prediction of Postoperative Patient-Reported Outcomes following Chiari Decompression Surgery
}

Shawyon Baygani, BS, Kristin Zieles,BS, and Andrew Jea, MD, MHA

Section of Pediatric Neurosurgery, Riley Hospital for Children, Department of Neurological Surgery, Indiana University School of Medicine, Indianapolis, Indiana

\section{Background and Hypothesis:}

Chiari malformation type I (CM-I) is a common pediatric disorder in which the cerebellar tonsils herniate the foramen magnum with associated spinal symptoms. The purpose of this study is to determine if the preoperative Pediatric Quality of Life Inventory (PedsQL) score is predictive of intermediate-term PedsQL outcomes following Chiari decompression surgery. The utility of preoperative patient-reported outcomes (PROs) to postoperative PROs has been previously demonstrated in adult spine surgery. However, to our knowledge, there is currently no widely accepted tool to predict short-, intermediate-, or long-term outcomes after pediatric Chiari decompression surgery.

\section{Project Methods:}

A prospectively maintained database was retrospectively reviewed. Patients who underwent first-time decompression for symptomatic Chiari malformation were identified and grouped by their preoperative PedsQL scores: mild disability (score 80-100), moderate disability (score 6080); and severe disability (score < 60). PedsQL outcomes were collected at 6-week, 3-month, and 6-month follow-up. Preoperative PedsQL subgroups were tested for an association with demographic, perioperative characteristics, and improvements between groups by one-way ANOVA, chi-square, or Wilcoxon signed rank test, where appropriate.

\section{Results:}


The postoperative patient- and parent-reported PedsQL values were significantly different between all three groups at 6-weeks, 3-months, and 6-months after surgery $(\mathrm{p}<0.05)$, except at the 6-month timepoint for parent-reported outcomes $(\mathrm{p}=0.111)$. Patients with higher preoperative disability demonstrated statistically significant greater improvements (compared to preoperative score) in parent-reported PedsQL at all timepoints after surgery, except at the 6month parent-reported outcomes $(\mathrm{p}=0.133)$.

\section{Conclusion:}

Patients with worse preoperative disability, as assessed by PedsQL, experienced lower absolute PedsQL scores at all timepoints after surgery but had greater improvement in short- and intermediate-term PROs. We conclude that PedsQL is an efficient and accurate tool that can quickly assess patient disability in the preoperative period and predict both short-term and longterm surgical outcomes. 